\title{
The Speed of Simple Random Walk and Anchored Expansion on Percolation Clusters: an Overview
}

\author{
Dayue Chen ${ }^{1 \dagger}$ and Yuval Peres ${ }^{2 \ddagger}$ \\ ${ }^{1}$ School of Mathematical Sciences, Peking University, Beijing, 100871, China, dayue@math.pku.edu.cn \\ ${ }^{2}$ Department of Statistics, University of California, Berkeley, CA 94720, USA, peresestat . berkeley.edu
}

\begin{abstract}
Benjamini, Lyons and Schramm (1999) considered properties of an infinite graph $G$, and the simple random walk on it, that are preserved by random perturbations. To address problems raised by those authors, we study simple random walk on the infinite percolation cluster in Cayley graphs of certain amenable groups known as "lamplighter groups". We prove that zero speed for random walk on a lamplighter group implies zero speed for random walk on an infinite cluster, for any supercritical percolation parameter $p$. For $p$ large enough, we also establish the converse. We prove that if $G$ has a positive anchored expansion constant then so does every infinite cluster of independent percolation with parameter $p$ sufficiently close to 1 ; We also show that positivity of the anchored expansion constant is preserved under a random stretch if, and only if, the stretching law has an exponential tail.
\end{abstract}

Keywords: Cayley graphs, percolation, random walks, speed, anchored expansion constant.

\section{Introduction}

Denote by $V(\mathbb{G})$ and $E(\mathbb{G})$, respectively, the sets of vertices and edges of an infi nite graph $\mathbb{G}$. In $p$ Bernoulli bond percolation in $\mathbb{G}$, each edge of $\mathbb{G}$ is independently declared open with probability $p$ and closed with probability $1-p$. Thus a bond percolation $\omega$ is a random subset of $E(\mathbb{G})$. We usually identify the percolation $\omega$ with the subgraph of $\mathbb{G}$ consisting of all open edges and their end-vertices. A connected component of this subgraph is called an open cluster, or simply a cluster. The probability that there is an infi nite cluster is monotone in $p$. Let $p_{c}=p_{c}(\mathbb{G})=\inf \{p$ : there is an infi nite cluster a.s. $\}$. When $p \in\left(p_{c}, 1\right)$, with positive probability the open cluster $\mathbb{H}$ that contains a fi xed vertex $o$ is infi nite.

Grimmett, Kesten and Zhang (1993) showed that simple random walk on the infi nite cluster of supercritical Bernoulli percolation in $\mathbb{Z}^{d}$ is transient for $d \geq 3$; in other words, in Euclidean lattices, transience is preserved when the whole lattice is replaced by the infi nite percolation cluster. Benjamini, Lyons and Schramm (1999), abbreviated as BLS (1999) hereafter, initiated a systematic study of the properties of a transitive graph $\mathbb{G}$ that are preserved under random perturbations such as passing from $\mathbb{G}$ to an infi nite

\footnotetext{
$\dagger$ supported by grant G1999075106 from the Ministry of Science and Technology of China.

¥supported by NSF grant DMS-0104073 and by a Miller Professorship at UC Berkeley.
} 
percolation cluster. They conjectured that positivity of the speed $\lim _{n}\left|X_{n}\right| / n$ for simple random walk $\left\{X_{n}\right\}$ is preserved, where $|x|$ is the graph distance from $x$ to $o$.

Conjecture (BLS(1999), Conjectures 1.4 and 1.5). If $\mathbb{G}$ is a Cayley graph on which simple random walk has positive speed, then a.s., simple random walk on each infinite cluster of p-Bernoulli percolation has positive speed.

If $\mathbb{G}$ is a Cayley graph on which simple random walk has zero speed, then a.s., simple random walk on every cluster of Bernoulli percolation also has zero speed.

For $S \subset V(\mathbb{G})$, denote by $|S|$ the cardinality of $S$ and by $\partial S=\partial_{\mathbb{G}} S$ the set of edges that have one end in $S$ and the other in $S^{c}$. A graph $\mathbb{G}$ is amenable if there exists a sequence $\left\{S_{n}\right\}$ of fi nite subsets of $V(\mathbb{G})$ such that $\lim _{n}\left|\partial S_{n}\right| /\left|S_{n}\right|=0$. We say that the graph $\mathbb{G}$ has sub-exponential growth if

$$
\limsup |\{x \in V(\mathbb{G}):|x| \leq n\}|^{1 / n}=1 .
$$

In particular, a graph that has sub-exponential growth is amenable. When $\mathbb{G}$ is an amenable Cayley graph, Burton and Keane (1989) show that $p$-Bernoulli percolation has a.s. at most one infi nite cluster. Theorem 1.3 of BLS (1999) states that if the Cayley graph $\mathbb{G}$ is non-amenable, then simple random walk on an infi nite cluster of Bernoulli percolation on $\mathbb{G}$ has positive speed. On the other hand, if a graph $\mathbb{G}$ has sub-exponential growth, i.e., if lim sup $|\{x \in V(\mathbb{G}):|x| \leq n\}|^{1 / n}=1$, then simple random walk on $\mathbb{G}$ (and on any subgraph) has zero speed (Varopoulos (1985)). It is therefore natural to study, as suggested in BLS (1999), a simple random walk on the infi nite cluster of an amenable Cayley graph with exponential growth. We address this problem and Theorems 2.1 and 2.2 below lend further support to this conjecture.

The lamplighter groups $G_{d}$, introduced in Kaimanovich and Vershik (1983), are amenable groups with exponential growth. The corresponding Cayley graphs $\mathbb{G}_{d}$ can be described as follows. A vertex of $\mathbb{G}_{d}$ can be identifi ed as $(m, \eta) \in \mathbb{Z}^{d} \times\left\{\right.$ fi nite subsets of $\left.\mathbb{Z}^{d}\right\}$. Heuristically, $\mathbb{Z}^{d}$ is the set of lamps, $\eta$ is the set of lamps which are on, and $m$ is the position of the lamplighter, or "marker". In each step of random walk, either the lamplighter switches the current lamp (from on to off, or from off to on) or moves to one of the neighboring sites in $\mathbb{Z}^{d}$. Each vertex of $\mathbb{G}_{d}$ has degree $2 d+1$; one edge corresponds to flipping the state of the lamp at location $m$, and the other $2 d$ edges correspond to moving the marker. For example, if $d=1$, the neighbors of $(m, \eta)$ are $(m+1, \eta),(m-1, \eta)$ and $(m, \eta \Delta\{m\})$, where $\eta \Delta\{m\}$ is $\eta \backslash\{m\}$ if $m \in \eta$, and is $\eta \cup\{m\}$ if $m \notin \eta$. For more details, see Lyons, Pemantle and Peres (1996). Kaimanovich and Vershik (1983) showed that simple random walk on $\mathbb{G}_{d}$ has speed zero for $d=1,2$ and has positive speed for $d \geq 3$.

\section{Results}

We now study simple random walk $\left\{X_{n}\right\}$ on the unique infi nite cluster of $p$-Bernoulli bond percolation in $\mathbb{G}_{d}$, starting from the fi xed vertex $o$. If $x$ is a vertex of the open cluster containing $o$, let $|x|_{\omega}$ be the graph distance in this cluster from $x$ to $o$.

Theorem 2.1 Let $d \in\{1,2\}$. Then the simple random walk on the infinite cluster of $\mathbb{G}_{d}$ has zero speed, i.e., $\lim _{n}\left|X_{n}\right|_{\omega} / n=0 \quad$ a.s.

Theorem 2.2 Suppose that $d \geq 3$. If $p>p_{c}\left(\mathbb{Z}^{d}\right)$, then the simple random walk on the infinite cluster of Bernoulli bond percolation in $\mathbb{G}_{d}$ has positive speed a.s. on the event that o is in the infinite cluster. 
These results can be extended further as follows.

Let $\mathbb{G}$ be the Cayley graph of a fi nitely generated infi nite group $\mathbf{G}$ with a fi xed, symmetric (i.e., closed under inversion) set of generators. We identify vertices of $\mathbb{G}$ with elements of the group G. Two points $x$ and $y$ of $\mathbb{G}$ are neighbors if $x y^{-1}$ is a generator.

Let $\mathbb{F}$ be the Cayley graph of a fi nite group $\mathbf{F}$ generated by a fi xed symmetric set of generators.

An element of $\sum_{x \in \mathbb{G}} \mathbb{F}$ is called a configuration and is denoted by $\eta=\{\eta(x): x \in V(\mathbb{G})\}$, where $\eta(x) \in$ $V(\mathbb{F})$ is the $x$-coordinate of $\eta$. In the following discussion, we only consider those $\eta$ 's for which $\eta(x)$ is the unit element of $\mathbf{F}$ for all but fi nitely many $x$ 's.

Defi ne a new graph $\mathbb{W}=\mathbb{G} \times \sum_{x \in \mathbb{G}} \mathbb{F}$ as a semi-direct product of $\mathbb{G}$ with the direct sum of copies of $\mathbb{F}$ indexed by $\mathbb{G}$. Vertices of $\mathbb{W}$ are identifi ed as $\left\{(m, \eta): m \in V(\mathbb{G}), \eta \in \sum_{x \in \mathbb{G}} \mathbb{F}\right\}$. Two vertices, $(m, \eta)$ and $\left(m_{1}, \xi\right)$, are neighbors if either

(i) $m=m_{1}, \eta(x)=\xi(x)$ for all $x \neq m$, and $\eta(m)$ is a neighbor of $\xi(m)$ in $\mathbb{F}$, or

(ii) $\eta=\xi$, and $m, m_{1}$ are neighbors in $\mathbb{G}$.

In particular, if $\mathbf{F}=\{0,1\}$ is the group of two elements and $\mathbb{G}$ is $\mathbb{Z}^{d}$, then $\mathbb{G} \times \sum_{x \in \mathbb{G}} \mathbb{F}$ is exactly the lamplighter group $\mathbb{G}_{d}$ described before Theorem 2.1 .

Suppose that $\mathbb{G}$ is amenable. Then the graph $\mathbb{G} \times \sum_{x \in \mathbb{G}} \mathbb{F}$ is amenable and grows exponentially. By Burton and Keane (1989), there is only one infi nite cluster when percolation occurs.

We say that $\mathbb{G}$ is recurrent if the simple random walk on $\mathbb{G}$ is recurrent; this is equivalent to $\mathbf{G}$ being a fi nite extension of $\mathbb{Z}^{1}$ or $\mathbb{Z}^{2}$. (see, e.g., Woess (2000), Theorem 3.24, p.36). The following theorem is a generalization of Theorem 2.1 .

Theorem 2.3 Suppose that $\mathbb{G}$ is a recurrent Cayley graph and that $\mathbb{F}$ is the Cayley graph of a finite group. Then the simple random walk on the infinite cluster of supercritical Bernoulli bond percolation in $\mathbb{W}=\mathbb{G} \times \sum_{x \in \mathbb{G}} \mathbb{F}$ has zero speed a.s.

On the other hand, if $\mathbb{G}$ is a transient Cayley graph, then for $p$ suffi ciently close to 1 , the infi nite cluster of $p$-Bernoulli bond percolation in $\mathbb{G}$ is transient. For $\mathbb{G}=\mathbb{Z}^{d}, d \geq 3$ and any $p>p_{c}(\mathbb{G})$ this is due to Grimmett, Kesten and Zhang (1993); for other Cayley graphs it is due to Benjamini and Schramm (1998), see also Theorem 9 in Angel, Benjamini, Berger and Peres (2002). The following theorem generalizes Theorem 2.2.

Theorem 2.4 Let $0<p<1$. Suppose that the infinite cluster of $p$-Bernoulli bond percolation on the Cayley graph $\mathbb{G}$ is transient and that $\mathbb{F}$ is the Cayley graph of a finite group. Then the simple random walk on the infinite cluster of $p$-Bernoulli bond percolation in $\mathbb{W}=\mathbb{G} \times \sum_{x \in \mathbb{G}} \mathbb{F}$ has positive speed a.s.

The proof of the above theorems can be found in Chen and Peres (2003). It should be emphasized that we do not know the answer of the following question even in lamplighter groups. "Does positive speed of simple random walk on a group imply positive speed on percolation clusters for ALL $p>p_{c}$ ?" (BLS(1999), Conjecture 1.4)

We now consider the stability of a related geometric quantity. We say that $S \subset V(\mathbb{G})$ is connected if the induced subgraph on $S$ is connected. Fix $o \in V(\mathbb{G})$. The anchored expansion constant of $\mathbb{G}$,

$$
\mathfrak{\imath}_{E}^{*}(\mathbb{G}):=\lim _{n \rightarrow \infty} \inf \left\{\frac{|\partial S|}{|S|}: o \in S \subset V(\mathbb{G}), S \text { is connected, } n \leq|S|<\infty\right\}
$$


was defi ned in BLS (1999). The quantity $i_{E}^{*}(\mathbb{G})$ does not depend on the choice of the basepoint $o$. It is related to the isoperimetric constant

$$
\mathfrak{\imath}_{E}(\mathbb{G}):=\inf \left\{\frac{|\partial S|}{|S|}: S \subset V(\mathbb{G}), S \text { is connected, } 1 \leq|S|<\infty\right\}
$$

but as we shall see, $\mathrm{t}_{E}^{*}(\cdot)$ is more robust. BLS $(1999)$ asked if the positivity of $\mathrm{t}_{E}^{*}(\mathbb{G})$ is preserved when $\mathbb{G}$ undergoes a random perturbation.

The importance of anchored expansion is exhibited by the following theorem, conjectured in BLS (1999). For a vertex $x$, denote by $|x|=|x|_{\mathbb{G}}$ the distance (the least number of edges on a path) from $x$ to the basepoint $o$ in $\mathbb{G}$.

Theorem 2.5 (Virág 2000) Let $\mathbb{G}$ be a bounded degree graph with $\mathrm{l}_{E}^{*}(\mathbb{G})>0$. Then the simple random walk $\left\{X_{n}\right\}$ in $\mathbb{G}$, started at o, satisfies $\liminf _{n \rightarrow \infty}\left|X_{n}\right| / n>0$ a.s. and there exists $C<\infty$ such that $\mathbf{P}\left[X_{n}=\right.$ $o] \leq \exp \left(-C n^{1 / 3}\right)$ for all $n \geq 1$.

Earlier, Thomassen (1992) showed that a condition weaker than $\mathrm{t}_{E}^{*}(\mathbb{G})>0$ suffi ces for transience of the random walk $\left\{X_{n}\right\}$. As noted in Virág (2000), in conjunction with Corollary 2.8, Theorem 2.5 implies that the speed of simple random walk on supercritical Galton-Watson trees is positive, a result fi rst proved in Lyons, Pemantle and Peres (1995). Other applications of anchored expansion are in Häggström, Schonmann and Steif (2000).

Theorem 2 of Benjamini and Schramm (1996) states that $p_{c}(\mathbb{G}) \leq 1 /\left(\mathfrak{l}_{E}(\mathbb{G})+1\right)$, but their proof yields the stronger inequality $p_{c}(\mathbb{G}) \leq 1 /\left(\mathrm{l}_{E}^{*}(\mathbb{G})+1\right)$.

Theorem 2.6 Consider $p$-Bernoulli percolation on a graph $G$ with $\mathrm{i}_{E}^{*}(G)>0$. If $p<1$ is sufficiently close to 1 , then almost surely on the event that the open cluster $\mathbb{H}$ containing o is infinite, we have $\mathrm{r}_{E}^{*}(\mathbb{H})>0$.

The analog of Theorem 2.6 for site percolation also holds. See the Appendix of Chen and Peres (2003). The proof of the theorem, given in Chen and Peres (2003), shows that the conclusion holds for all $p>$ $1-h /(1+h)^{1+\frac{1}{h}}$ where $h=\mathrm{l}_{E}^{*}(\mathbb{G})$. A refi nement of the argument, due to Gábor Pete, shows that the conclusion holds for all $p>1 /\left(\mathfrak{l}_{E}^{*}(\mathbb{G})+1\right)$. Again, Theorem 2.6 only partially answers Question 6.5 of BLS(1999): "Does nonamenability of a group imply anchored expansion on percolation clusters for ALL $p>p_{c}$ ?"

Next, let $\mathbb{G}$ be an infi nite graph of bounded degree and pick a probability distribution $v$ on the positive integers. Replace each edge $e \in E(\mathbb{G})$ by a path that consists of $L_{e}$ new edges, where the random variables $\left\{L_{e}\right\}_{e \in E(\mathbb{G})}$ are independent with law $v$. Let $\mathbb{G}^{v}$ denote the random graph obtained in this way. We call $\mathbb{G}^{v}$ a random stretch of $\mathbb{G}$. If the support of $v$ is unbounded, then $\imath_{E}\left(\mathbb{G}^{v}\right)=0$ a.s. Say that $v$ has an exponential tail if $\mathrm{v}[\ell, \infty)<e^{-\varepsilon \ell}$ for some $\varepsilon>0$ and all suffi ciently large $\ell$.

Theorem 2.7 Suppose that $\mathbb{G}$ is an infinite graph of bounded degree and $\mathrm{t}_{E}^{*}(\mathbb{G})>0$. If $\mathrm{v}$ has an exponential tail, then $\mathrm{l}_{E}^{*}\left(\mathbb{G}^{v}\right)>0$ a.s.

On the other hand, if $v$ has a tail that decays slower than exponentially, then for any $c>E L$ and any $\varepsilon>0$, we have $P\left(\sum_{i=1}^{n} L_{i} \geq 2 c n\right) \geq e^{-\varepsilon n}$ for suffi ciently large $n$, where $\left\{L_{i}\right\}$ are i.i.d. with law $v$. Let $\mathbb{G}$ be 
a binary tree with the root $o$ as the basepoint. Pick a collection of $2^{n}$ pairwise disjoint paths from level $n$ to level $2 n$.

$$
P\left(\text { along at least one of these } 2^{n} \text { paths } \sum_{i=1}^{n} L_{i} \geq 2 c n\right) \geq 1-\left(1-e^{-\varepsilon n}\right)^{2^{n}} \geq 1-\exp \left(-e^{-\varepsilon n} 2^{n}\right) \rightarrow 1 .
$$

With probability very close to 1 (depending on $n$ ) there is a path from level $n$ to level $2 n$ along which $\sum_{i=1}^{n} L_{i} \geq 2 c n$. Take such a path and extend it to the root $o$. Let $S$ be the set of vertices in the extended path from the root $o$ to level $2 n$. Then

$$
\frac{|\partial S|}{\sum_{e \in E(S)} L_{e}} \leq \frac{2(2 n+1)}{2 c n} \approx \frac{2}{c} .
$$

Since $c$ can be arbitrarily large, $\mathrm{l}_{E}^{*}\left(\mathbb{G}^{v}\right)=0$ a.s. This shows that the exponential tail condition is necessary to ensure the positivity of $\mathrm{\imath}_{E}^{*}\left(\mathbb{G}^{v}\right)$.

By a Galton-Watson tree we mean a family tree of a Galton-Watson process.

Corollary 2.8 For a supercritical Galton-Watson tree $\mathbb{T}$, given non-extinction, we have $\mathrm{1}_{E}^{*}(\mathbb{T})>0$ a.s.

Theorem 2.7 and Corollary 2.8 answer Questions 6.3 and 6.4 of BLS (1999). Proofs of Theorem 2.7 and Corollary 2.8 can be found in Chen and Peres (2003).

\section{References}

[1] Angel, O., Benjamini, I., Berger, N. and Peres, Y., (2002) Transience of percolation clusters on wedges, Preprint.

[2] Benjamini, I., Lyons, R. and Schramm, O. (1999) Percolation Perturbations in Potential Theory and Random Walks, in Random walks and Discrete Potential Theory. Cambridge Univ. Press, 56-84.

[3] Benjamini, I. and Schramm, O. (1996) Percolation beyond $\mathbb{Z}^{d}$, many questions and a few answers, Electronic Commun. Probab. 1, 71-82.

[4] Benjamini, I. and Schramm, O. (1998) Oriented random walk on the Heisenberg group and percolation. Unpublished manuscript.

[5] Burton, R.M. and Keane, M. (1989) Density and uniqueness in percolation, Commun. Math. Phys. 121, 501-505.

[6] Chen, D. and Peres, Y. (2003) Anchored Expansion, Percolation and Speed, with an appendix by Gábor Pete, to appear Ann. Probab.

[7] Grimmett, G.R., Kesten, H. and Zhang, Y. (1993) Random walk on the infi nite cluster of the percolation model, Probab. Th. Rel. Fields, 96, 33-44.

[8] Häggström, O., Schonmann, R. and Steif, J. (2000) The Ising model on diluted graph and strong amenability. Ann. Probab., 28, 1111-1137. 
[9] Kaimanovich, V. A. and Vershik, A. M. (1983) Random walks on discrete groups: boundary and entropy, Ann. Probab., 11, 457-490.

[10] Lyons, R., Pemantle, R. and Peres, Y. (1995) Ergodic theory on Galton-Watson trees: speed of random walk and dimension of harmonic measure, Ergodic Theory Dynamical Systems, 15, 593619.

[11] Lyons, R., Pemantle, R. and Peres, Y. (1996) Random walks on the lamplighter group. Ann. Probab. 24, 1993-2006.

[12] Thomassen, C. (1992) Isoperimetric inequalities and transient random walks on graphs, Ann. Probab., 20, 1592-1600.

[13] Varopoulos, N. Th. (1985) Long range estimates for Markov chains, Bull. Soc. Math., 2éme serie 109, 113-119.

[14] Virág, B. (2000) Anchored expansion and random walk, Geom. Func. Anal. 10, 1588-1605.

[15] Woess, W. (2000) Random Walks on Infinite Graphs and Groups. Cambridge Univ. Press. 